\title{
The Robustness of Self-Organized Zonal Jets in Unforced, Turbulent Vorticity Fields
}

\author{
James Y.-K. Cho and Lorenzo M. Polvani \\ Program in Applied Mathematics and Department of Applied Physics \\ Columbia University, New York, NY 10027, USA
}

\begin{abstract}
A wide parameter space is spanned to check the influence of numerical parameters on selforganized jets in rotating, turbulent shallow-water flows. It is found that, even in harsh physical (strongly ageostrophic) and numerical (filtered, hyperviscous, high-resolution) environments, the basic morphogenesis of steady jets, qualitatively reflecting the Rhines scale, is robust. However, detailed features of the jet pattern, such as the amplitude, the width, and the location can be substantially affected by numerical parameters for a fixed set of physical parameters. In general, while the amount of energy lost seems to be a good predictor of maximum jet amplitude and width, a nonmonotonic dependence of these features on the numerical parameters is observed. The most acute sensitivity is associated with the use of a $\nabla^{16}$ hyperdissipation operator in conjunction with a Robert-Asselin filter, which leads to substantial meridional shifts of the entire jet pattern as the viscosity coefficient is varied.
\end{abstract}

\section{Introduction}

Zonal structures in 2D, incompressible homogeneous turbulence can arise in the presence of $\beta$, the planetary vorticity gradient [5]. In past studies, the $\beta$-effect with forcing has been shown to provide an anisotropic arrest mechanism for the inversely cascading energy spectrum [8]. Starting from an initially random flow field, this arrest leads to a steady, multiple zonal jet pattern, qualitatively reflecting the scale of arrest-called the Rhines scale, $L_{\beta}$.

Recently, we have performed freely-evolving, shallow-water (i.e., 2D, compressible) turbulence simulations in full spherical geometry in order to discriminate between the robust spontaneous features of the flow from those which are forcibly generated or sustained [1]. In that work, it was demonstrated that the jet formation phenomenon can be extended beyond the simple $\beta$-plane to the more complex spherical geometry and that forcing is not a necessary component for the formation of robust, steady, quasi-zonal jets. Furthermore, the self-organizing steady jets were found to be a physically general phenomenon since they emerged in a wide range of physical parameter values (i.e., rotation rate, radius, scale height, and initial energy distribution) ${ }^{1}$.

In this work, we validate the robustness of jet-formation in a series of simulations performed under a wide variety of numerical situations. Under a fixed set of physical parameters (e.g., identical rotation rate, radius, scale height, total energy, and balanced initial state), the independent effects of 1) resolution, 2) initial peak-energy scale, 3) hyperdissipation, 4) viscosity coefficient, 5) RobertAsselin filter, 6) semi-implicit time-stepping scheme, and 7) timestep-size are studied.

\footnotetext{
${ }^{1}$ The only requirement seems to be a finite deformation radius smaller than approximately $1 / 3$ of the sphere's radius.
} 


\section{Numerical procedure and the basic profile}

The shallow-water equations, which we are directly integrating are:

$$
\begin{aligned}
\partial_{t} \zeta_{a} & =-\frac{1}{a\left(1-\mu^{2}\right)} \partial_{\lambda}\left(\tilde{U} \zeta_{a}\right)-\frac{1}{a} \partial_{\mu}\left(\tilde{V} \zeta_{a}\right)+\hat{\mathcal{D}} \zeta \\
\partial_{t} \delta & =\frac{1}{a\left(1-\mu^{2}\right)} \partial_{\lambda}\left(\tilde{V} \zeta_{a}\right)-\frac{1}{a} \partial_{\mu}\left(\tilde{U} \zeta_{a}\right)-\nabla^{2}\left[g h^{\prime}+\frac{\tilde{U}^{2}+\tilde{V}^{2}}{2\left(1-\mu^{2}\right)}\right]+\hat{\mathcal{D}} \delta \\
\partial_{t} h^{\prime} & =-\frac{1}{a\left(1-\mu^{2}\right)} \partial_{\lambda}\left(\tilde{U} h^{\prime}\right)-\frac{1}{a} \partial_{\mu}\left(\tilde{V} h^{\prime}\right)-\delta+\hat{\mathcal{D}} h^{\prime}
\end{aligned}
$$

where

$$
\begin{aligned}
\zeta_{a} & \equiv \frac{1}{a\left(1-\mu^{2}\right)} \partial_{\lambda} \tilde{V}-\frac{1}{a} \partial_{\mu} \tilde{U}+2 \Omega \mu \\
\delta & \equiv \frac{1}{a\left(1-\mu^{2}\right)} \partial_{\lambda} \tilde{U}+\frac{1}{a} \partial_{\mu} \tilde{V}
\end{aligned}
$$

and $h^{\prime} \equiv h-H$ are the three prognostic variables, representing the absolute vorticity, the divergence and the difference of the total and the average surface heights, respectively, in $(\lambda, \vartheta)=$ (longitude, latitude) coordinates ${ }^{2}$. The pair, $(\tilde{U}, \tilde{V}) \equiv(u, v) \cdot \sqrt{1-\mu^{2}}$, are the scaled zonal and meridional velocities, respectively; and, $\hat{\mathcal{D}}$ is the hyperdissipation operator, $(-1)^{p+1} \nu_{2 p} \nabla^{2 p}$. The physical parameters, $a, g$, and $\Omega$ are the radius, the local gravitational acceleration, and the rotation rate, respectively. Recall that Eqs. 1-3 imply the material conservation of potential vorticity, $q \equiv \zeta_{a} / h$, in the limit of infinite Reynolds number.

A standard pseudospectral algorithm [2] which expands the prognostic variables in a series of spherical harmonics with a triangular-shaped truncation in $(m, n)$ spectral space is employed ${ }^{3}$. The hyperviscosity is applied in spectral space and acts as a variable band-pass filter; it is a commonlyused tool in numerical computations to increase the effective Reynolds number. The viscosity coefficient, $\nu_{2 p}$, is initially chosen in a series of runs such that $\nu_{2 p}\left[\frac{n(n+1)}{a^{2}}\right]^{p}=1$ and then reduced in subsequent runs until, due to the lack of dissipation, the run succumbs to a pile-up at the farrange of the energy spectrum. For the timestepping, a second-order accurate leapfrog scheme with a time-lagged (implicit) procedure for the elliptic, viscosity portions of the equations is used. In this work, we also consider the effects of the commonly-used Robert-Asselin filter [7], as well as a scheme to semi-implicitly treat Eqs. 2 and $3[6]$, which often allows a substantial savings in the number of required timesteps.

In order to establish that the appearance of zonal jets is a robust behavior and is insensitive to the many numerical parameters that need to be chosen, we have selected a particularly "difficult-to-simulate" set of physical parameters-that is, one with high initial energy and very ageostrophic initial state. The physical parameters are: $(a, \Omega, g, H)=\left(2.48 \times 10^{7} \mathrm{~m}, 9.77 \times\right.$ $10^{-5} \mathrm{~s}^{-1}, 11.1 \mathrm{~m} / \mathrm{s}, 40.0 \times 10^{3} \mathrm{~m}$ ); the initial energy distribution corresponds to a spatially averaged velocity, $\left\langle u^{2}+v^{2}\right\rangle^{\frac{1}{2}}=370 \mathrm{~m} / \mathrm{s}$ and a narrow spectral peak about the centroid value, $\bar{n}$. The varied numerical parameters for all the runs discussed in this paper are tabulated in Table 1.

Figure 1 illustrates a typical, long-time (300 rotations of the sphere) potential vorticity evolution (a) and the corresponding zonal jet profile (b) of Run A2 in Table 1. Note the characteristic pattern of the three, steady east-west jets (marked with arrows), corresponding to the extrema of meridional

\footnotetext{
${ }^{2}$ The variable, $\mu \equiv \sin \vartheta$, is the axial height.

${ }^{3}$ Following the standard notation, $m$ and $n$ are the zonal and total wavenumbers, respectively.
} 
$q$ gradient, and the pronounced equatorial jet which quickly emerge. The key result of this study is that, for a given set of physical parameters, the basic morphogenesis of jets is relatively insensitive to numerical parameter variations, while the precise jet amplitude, width, and location may be substantially affected. In the following section, using this run as a reference run, we describe and compare the variations from this basic pattern under a wide variety of numerical conditions.

\section{Parameter variations}

\subsection{Resolution (Runs R1 to R3)}

Figure 2 shows the dependence of jets on the resolution; the "T"-number refers to the number of resolved Fourier modes and is equal to $\frac{1}{3}(2 N-1)$, where $\mathrm{N}$ is the number of physical grid points in the meridional direction. Note that, while the basic behavior is not affected, the jet amplitudes decrease with coarser resolution. The main reason for this is the amount of dissipation suffered in each case: $19 \%, 17 \%$, and $12 \%$ of the initial energy for T21, T42, and T85 runs, respectively.

It is worth mentioning that, at T85 resolution, the physical parameters are such that both the $L_{\beta}$ and the $L_{D}$ scales are well-resolved and separated $\left(L_{\beta} / L_{D} \approx 10\right)$ and that here the Rhines length, which is of the order of the observed jet scale, clearly sets the jet scale rather than the deformation radius ${ }^{4}$. In the presence of strong $\beta$, the vortices may be quickly sheared out (cf. Fig. $1(\mathrm{a}))$ in contrast to the longevity experienced in the absence of $\beta^{5}$. In the simulations presented here, the shearing of vortices provide an unimpeded inverse cascade past the $L_{D}$ scale to the $L_{\beta}$ scale, as will be shown in the next set of variations.

\subsection{Initial centroid location (Runs N1 to N3)}

Figure 3 shows the effects of beginning the simulation with the peak-energy set at different scales (here, quantified by the energy centroid, $\bar{n} \equiv \sum_{n} n E(n) / \sum_{n} E(n)$, where $E(n)$ is the energy density).

First, this set of variations indicates that, as in the resolution variation, the amount of dissipation is heavily influencing the basic profile. There is a $12.2 \%, 12.2 \%, 13.4 \%$, and $14.5 \%$ energy-loss in the runs corresponding to the maximum amplitudes of $-250 \mathrm{~m} / \mathrm{s},-231 \mathrm{~m} / \mathrm{s},-162 \mathrm{~m} / \mathrm{s}$, and $-50 \mathrm{~m} / \mathrm{s}$, respectively; for the first two cases, the energy-loss difference is not detectable, but the difference in the loss of potential-enstrophy $\left(=\left\langle\frac{1}{2} \zeta_{a}^{2} h\right\rangle\right)$ is significant: only $56 \%$ in the former and 93\% in the latter. Also, the jets of the more dissipative cases are narrower.

Second, there also seems to be an "extra" jet near the poles of the runs with centroid 18 and 36 in Fig. 3. This is the result of a strong coherent vortex that just happens to be centered at the pole, an event attributed to the moderate value of $L_{D} / a$ which is 0.14 in these runs [1]). A much more prevalent situation is where such a vortex is off-centered or is not formed at all. Another general observation about jets on the sphere is that, due to the "spiraling" and the general "wavy" appearance of the $q$ bands (see, for example, Fig. 1(a)), the zonally averaged profile is much less sharp than an unaveraged profile.

Finally, we note that although the basic 3 -jet pattern does not seem to depend on the initial energy-centroid value, this study does not show a simple behavior between the jet amplitude and the peak-scale. That is, we do not observe a monotonic relationship between the two quantities, as might be expected from the energy-loss characteristics of turbulence with increased $\bar{n}$-namely,

\footnotetext{
${ }^{4}$ Accordingly, there is no secondary peak corresponding to $L_{D}$ in the energy spectrum.

${ }^{5}$ In the situation without $\beta, L_{D}$ provides the arrest-mechanism and controls the scale of the surviving coherent vortices [4].
} 
more dissipation. Furthermore, the deviation from monotonicity is not unique to this set of parameter variation but also characterizes other sets of, variations, as will be shown in the following subsections.

\subsection{Hyperviscosity (Runs H1 to H3)}

Figure 4 shows the results obtained using three separate dissipation operators. Here again, the basic pattern is unchanged and the energy-loss provides a good measure of the jet amplitudes, with $22.2 \%, 12.2 \%$, and $13.8 \%$ for the $2 p=2,6$, and 16 runs, respectively. The more dissipative runs have slightly narrower jets.

As alluded to in Section 2, hyperviscosity is normally used to confine the dissipation to smaller scales, reducing the total dissipation. Following this, a larger amplitude jet would naively be expected for the larger $2 p$ value. However, as observed in the previous subsection (centroid location variation), this expectation is not born out, and there seems to be a more complicated interaction between the hyperviscosity and the other numerical parameters affecting the flow.

For example, the spectrum for the $2 p=16$ case show more energy in small scales, as expected. In physical space, this is reflected by the lack of strong, $m=1$ mode band of $q$-contours at mid-latitude, as is in the $2 p=6$ case (cf. Fig. 1(a)); instead, there is a weak band of large $m$ mode. We point out here that we are using a Robert-Asselin filter, to allow integration of a strongly ageostrophic field. It is known that this filter adds a significant amount of dispersive error; therefore, there exist a possibility of an excessive amount of underdamped, small-scale (high-frequency) structures contributing to a lack of coherence in the physical field. Hence, it would seem that in our calculations an ultra-high hyperdissipation may actually present a hindrance to the formation of a strong jet ${ }^{6}$. This then raises the interesting question of whether the viscosity coefficient, $\nu_{2 p}$, can be used to modify the situation perhaps by offering a modified dissipation characteristics to the spectrum, and therefore we now turn to the variation of that parameter with $2 p$ set at 16 .

\subsection{Viscosity coefficient (Runs V1 to V3)}

In Section 2, the procedure for choosing $\nu_{2 p}$ was outlined; the procedure allows the most inviscid simulation to be performed given all the other numerical parameters. Here, we are fixing the dissipation operator ( $2 p=16$ in all three cases) and varying the viscosity coefficient. Figure 5 shows the result. As can be seen, there is a consistent reduction in maximum amplitude with dissipations of $12.3 \%, 13.8 \%$, and $15.1 \%$ for the three increasing coefficient values.

Rather obvious in this set of variations is the large, random shifts in the jet locations. We note that in the runs reported in this paper, such shifts are sometimes observed when a different set of phases in the spectral modes are used (i.e., for this set of physical parameters, the jet location is sensitive to viscosity and initial flow field). We also note that, in spectral space, an increased coefficient value for the $2 p=16$ operator shaves off a wider strip of energy at the far-end of the spectrum (a lower $2 p$ value is far less selective and draconian). Since these runs possess identical initial phases (and every other parameters except $\nu_{2 p}$ ), the shifts here are due to the presence of undissipated, fine-scales. Such behavior suggest a strong coupling between another numerical tool that selectively alters small-scale features in these runs, the Robert-Asselin filter.

\footnotetext{
${ }^{6}$ See references [3] and [1] for additional hyperviscosity effects in $\beta=0$ situations.
} 


\subsection{Robert-Asselin filter (Runs A1 to A4)}

Figure 6 illustrates the effects of varying the amount of Robert-Asselin filtering. The Robert-Asselin filter is a temporal filter used to suppress "time-splitting" instability arising from the spurious computational mode associated with the leapfrog scheme. It is well-known that while damping the computational mode, this filter also damps the physical mode and increases the phase error. We point out here that, due to the complicated flow fields arising from the chosen set of physical parameters, no run presented in this paper could be integrated for more than 5 rotations in the absence of this filter before succumbing to instabilities inherent in the shallow-water system.

As can be seen from Fig. 6, the precise relationship between the filter and the jets is again not monotonic, though in all the cases the basic jet pattern is not affected. Below the 0.025 value, the simulation becomes unstable; above the 0.1 value, the simulation suffers large amounts of dissipation. The jet amplitudes in the figure can be grouped by their energy-losses: $12 \%$ for the runs with 0.050 and 0.100 values and $14 \%$ for the runs with 0.025 and 0.075 values. As stated previously, in this variation, in which $2 p=6$, there is no detectable shift in the jet locations. We also note that the jet widths are not affected, and the filter seems to be fairly harmless for this set of parameters.

\subsection{Semi-implicit timestepping (Runs S1 and S2)}

Figure 7 shows the effect of using a semi-implicit timestepping procedure. The used procedure separates the higher-frequency gravity modes from the lower-frequency Rossby modes and treats the gravity modes implicitly. This scheme usually allows a larger timestep to be taken. As can be seen, there is basically no difference in the jet pattern produced by the semi-implicit procedure; only a slight reduction in amplitude is visible, but no significant difference between the two runs is detected by any of the energy, potential enstrophy, energy and height centroids, skewness or kurtosis measures.

However, it is worth mentioning here that, although clearly advantageous in more balanced environments, in strongly ageostrophic situations (as in the runs presented here), the advantage is not fully realized since maximum velocities of the flow are quite close to the gravity wave speed, and hence the scale separation is small.

\subsection{Timestep-size (Runs D1 to D3)}

Finally, Fig. 8 shows the resulting jet patterns when the timestep size is increased. The figure again shows that the basic pattern is not affected. We note that the Robert-Asselin filter restricts the Courant number for stability to be slightly less than the "less-than-one" criterion valid for the unfiltered leapfrog scheme. The maximum Courant number reached during the evolution in each case is $0.29,0.58$, and 0.88 for $\Delta t$ values of 60,120 , and 180 secs, respectively. Here, too, the behavior is not one expected from a "textbook" analysis of filtered leapfrog scheme based on the linear, "oscillation" equation: $\partial_{t} \hat{\psi}=i \omega \hat{\psi}$, where $\hat{\psi}=\hat{\psi}(m, n, t)$ is the spectral amplitude and $\omega$ is a positive constant.

\section{Conclusion}

In this paper, a wide range of the numerical parameter space was spanned to check the influence of numerical parameters on self-organized jets in rotating, unforced shallow-water turbulence. It was found that, even in very harsh physical and numerical environments, the basic steady jet 
pattern qualitatively reflecting the Rhines scale is robustly formed - though detailed features such as the amplitude, the width, and the location of the jets can be substantially affected. In general, dissipation was found to be a good qualitative measure of the jet amplitude and width: more the dissipation, lower the amplitude and narrower the equatorial jet. In addition, while the energy-loss correlated well with those features, the jet profile behaviors were in general not monotonic with respect to the varied numerical parameters. The most blatant deviation consisting of substantial meridional shifts from the basic profile was observed when a $\nabla^{16}$ hyperdissipation operator was used, possibly indicating complex interaction between that operator and the Robert-Asselin filter required to integrate a highly ageostrophic flow field.

We conclude that the results of this paper, combined with the general appearance of robust jets in a wide range of physical parameters, robustly establish the phenomenon of spontaneous, steady jet formation in freely-evolving, shallow-water turbulent flows.

\section{Acknowledgments}

This research was supported in part by the NSF National Young Investigator Award to LMP. The Pittsburgh Supercomputing Center, supported by the NSF, provided the computing resources and lessons. The original version of global shallow-water code was provided by J. Hack and R. Jakob. The authors also benefited from the boundless enthusiasm of D. Dritschel and the generosity of M. DiBattista and thank L. Rivier for his assistance with the translation of the abstract.

\section{References}

[1] Cho, J. Y-K. and Polvani, L.M., "The Emergence of Jets and Vortices in a Freely Evolving, Shallow-Water Turbulence on a Sphere," Phys. Fluids, 8, pp. 1531, 1996.

[2] Hack, J.J. and Jakob, R., NCAR Technical Note NCAR/TN-343+STR, 1992.

[3] Mariotti, A., Legras, B. and Dritschel, D.G., "Vortex Stripping and the Erosion of Coherent Structures in Two-Dimensional Flows," Phys. Fluids, 6, pp. 3954, 1994.

[4] Polvani, L.M., McWilliams, J.C., Spall, M.A. and Ford, R., "Coherent Structures of Shallow-Water Turbulence: Deformation-Radius Effects, Cyclone/Anticyclone Asymmetry and Gravity-Wave Generation," Chaos, 4, pp. 177, 1994.

[5] Rhines, P.B., "Waves and Turbulence on a Beta Plane," J. Fluid Mech., 69, pp. 417, 1975.

[6] Ritchie, H., "Application of Semi-Lagrangian Method to a Spectral Method of the Shallow Water Equations," J. Atmos. Sci., 116, pp. 1587, 1988.

[7] Robert, A.J., "The Integration of a Low-Order Spectral Form of the Primitive Meteorological Equations," J. Meteor. Soc. Japan, 44, pp. 237, 1966.

[8] Vallis, G.K. and Maltrud, M.E., "Generation of Mean Flows and Jets on a Beta Plane and over Topography," J. Phys. Ocean., 23, pp. 1346, 1993 (and references therein). 
Table 1: Summary of Runs

\begin{tabular}{cccccccc}
\hline \hline Run & Trunc & $\bar{n}$ & $2 p$ & $\nu_{2 p}$ & $A F C$ & $S I T$ & $\Delta t($ sec $)$ \\
\hline \hline R1 & T21 & 14 & 6 & $5 \times 10^{29}$ & 0.050 & yes & 240 \\
R2 & T42 & 14 & 6 & $1 \times 10^{29}$ & 0.050 & yes & 120 \\
R3 & T85 & 14 & 6 & $5 \times 10^{28}$ & 0.050 & yes & 60 \\
\hline N1 & T85 & 5.5 & 6 & $5 \times 10^{28}$ & 0.050 & yes & 60 \\
N2 & T85 & 18 & 6 & $5 \times 10^{28}$ & 0.050 & yes & 60 \\
N3 & T85 & 25 & 6 & $5 \times 10^{28}$ & 0.050 & yes & 60 \\
N4 & T85 & 36 & 6 & $5 \times 10^{28}$ & 0.050 & yes & 60 \\
\hline H1 & T85 & 14 & 2 & $6 \times 10^{6}$ & 0.050 & yes & 60 \\
H2 & T85 & 14 & 6 & $5 \times 10^{28}$ & 0.050 & yes & 60 \\
H3 & T85 & 14 & 16 & $5 \times 10^{84}$ & 0.050 & yes & 60 \\
\hline V1 & T85 & 14 & 16 & $5 \times 10^{83}$ & 0.050 & yes & 60 \\
V2 & T85 & 14 & 16 & $5 \times 10^{84}$ & 0.050 & yes & 60 \\
V3 & T85 & 14 & 16 & $5 \times 10^{85}$ & 0.050 & yes & 60 \\
\hline A1 & T85 & 14 & 6 & $5 \times 10^{28}$ & 0.025 & yes & 60 \\
A2 & T85 & 14 & 6 & $5 \times 10^{28}$ & 0.050 & yes & 60 \\
A3 & T85 & 14 & 6 & $5 \times 10^{28}$ & 0.075 & yes & 60 \\
A4 & T85 & 14 & 6 & $5 \times 10^{28}$ & 0.100 & yes & 60 \\
\hline S1 & T85 & 14 & 6 & $5 \times 10^{28}$ & 0.050 & yes & 60 \\
S2 & T85 & 14 & 6 & $5 \times 10^{28}$ & 0.050 & no & 60 \\
\hline D1 & T85 & 14 & 6 & $5 \times 10^{28}$ & 0.050 & yes & 60 \\
D2 & T85 & 14 & 6 & $5 \times 10^{28}$ & 0.050 & yes & 120 \\
D3 & T85 & 14 & 6 & $5 \times 10^{28}$ & 0.050 & yes & 180 \\
\hline \hline
\end{tabular}




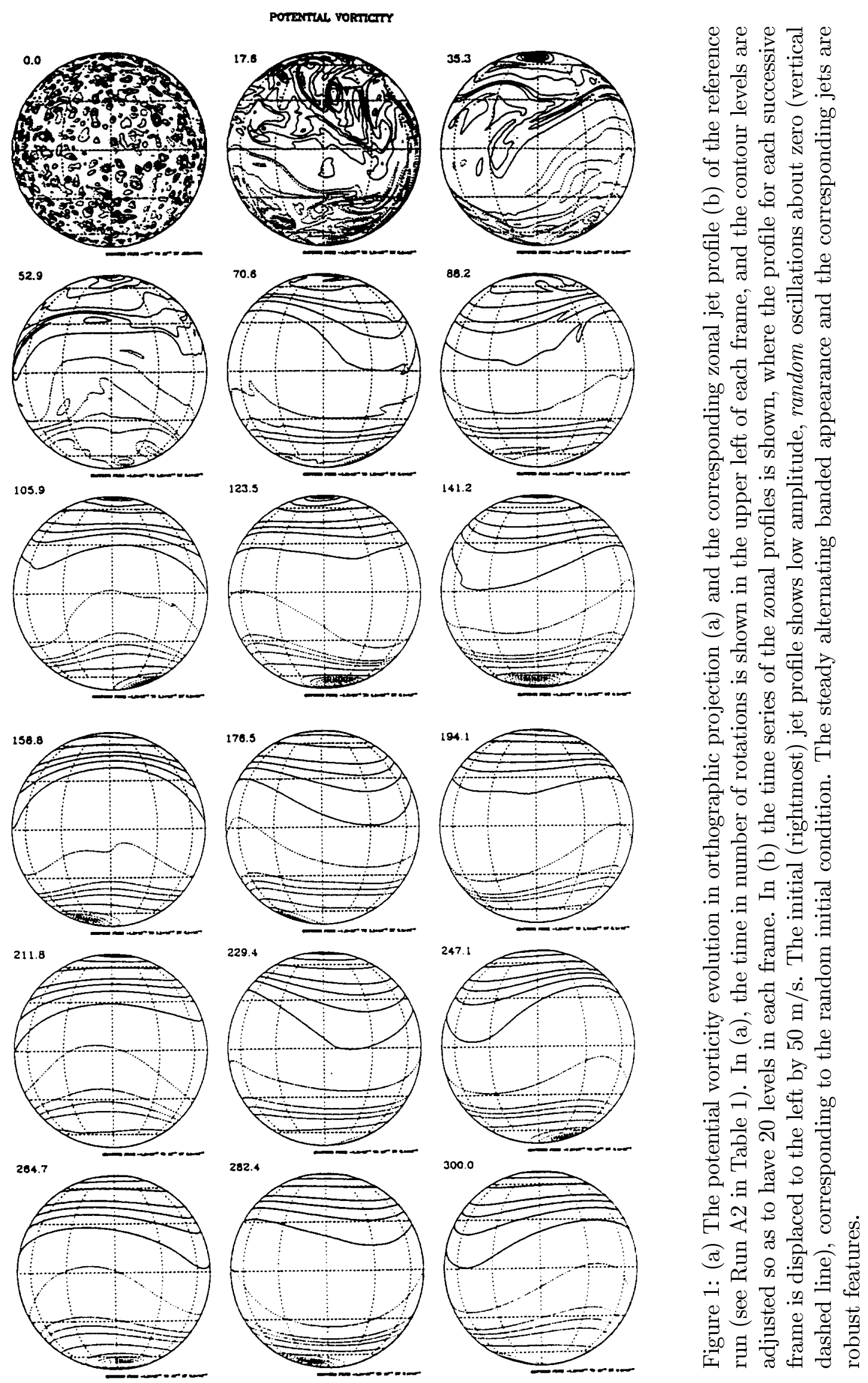




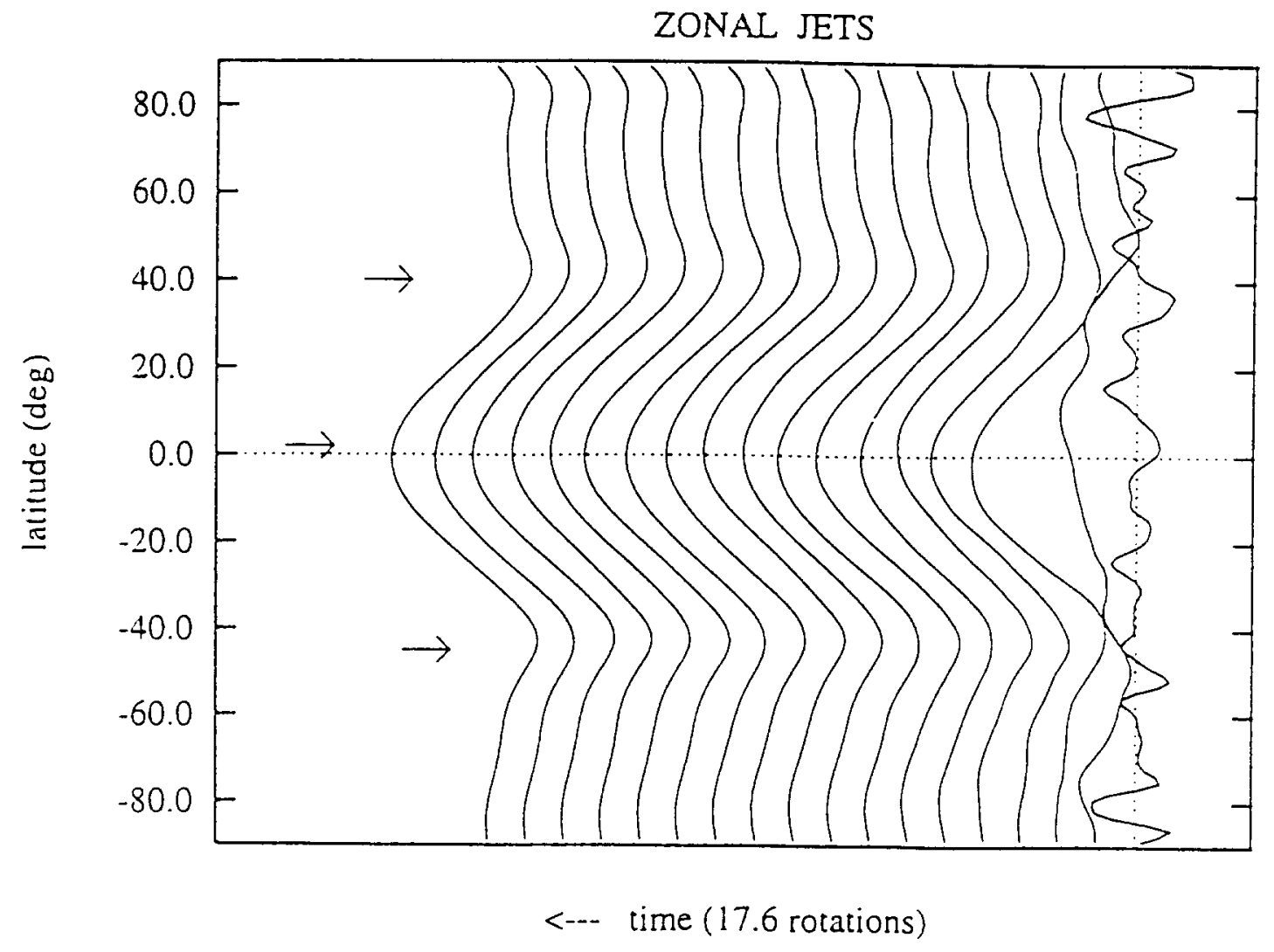

Figure 1: (b) 


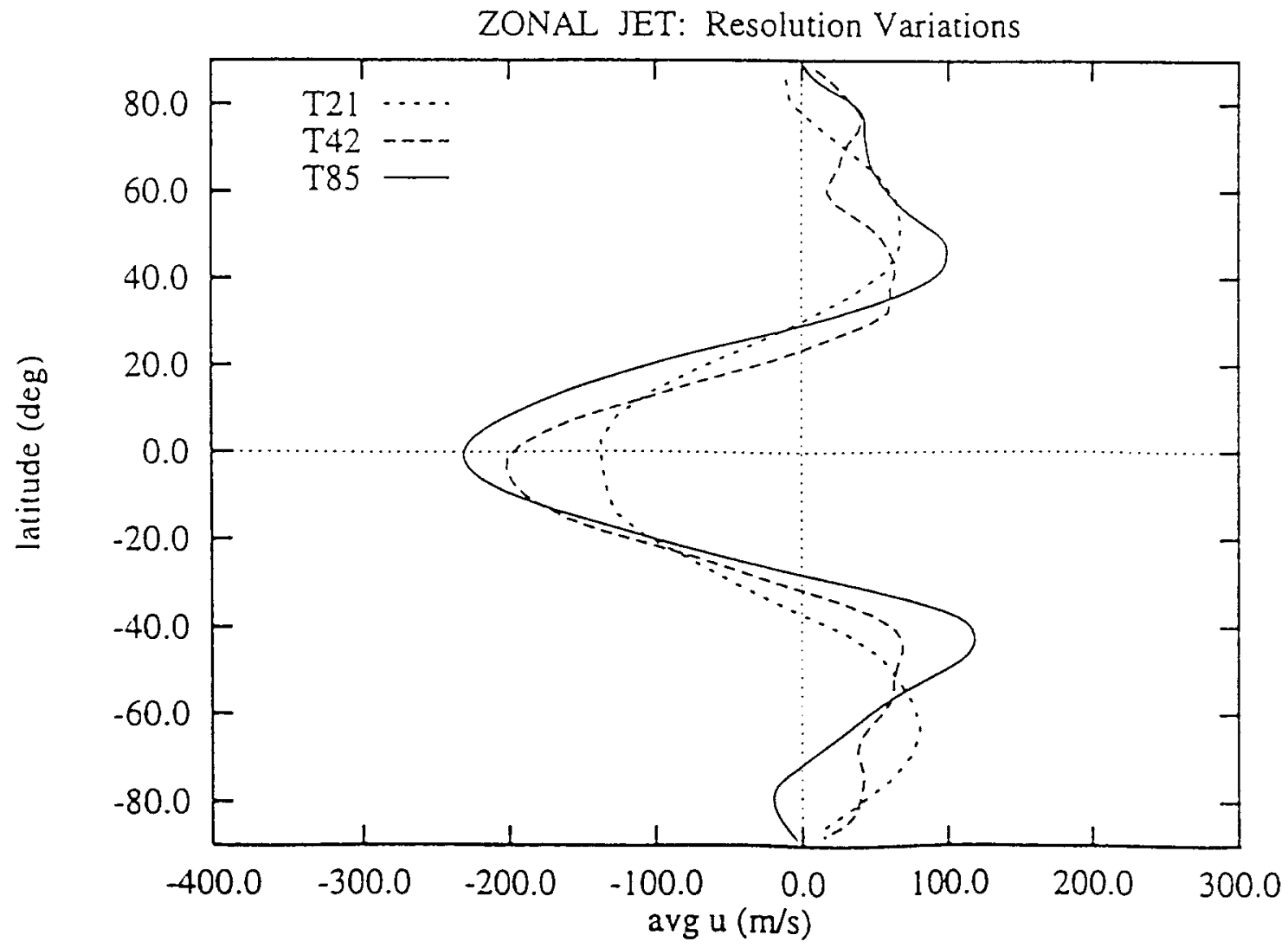

Figure 2: The zonal jet profile of three different resolutions (Runs R1 to R3 in Table 1). The "T" number refers to the number of resolved modes in the calculations. 


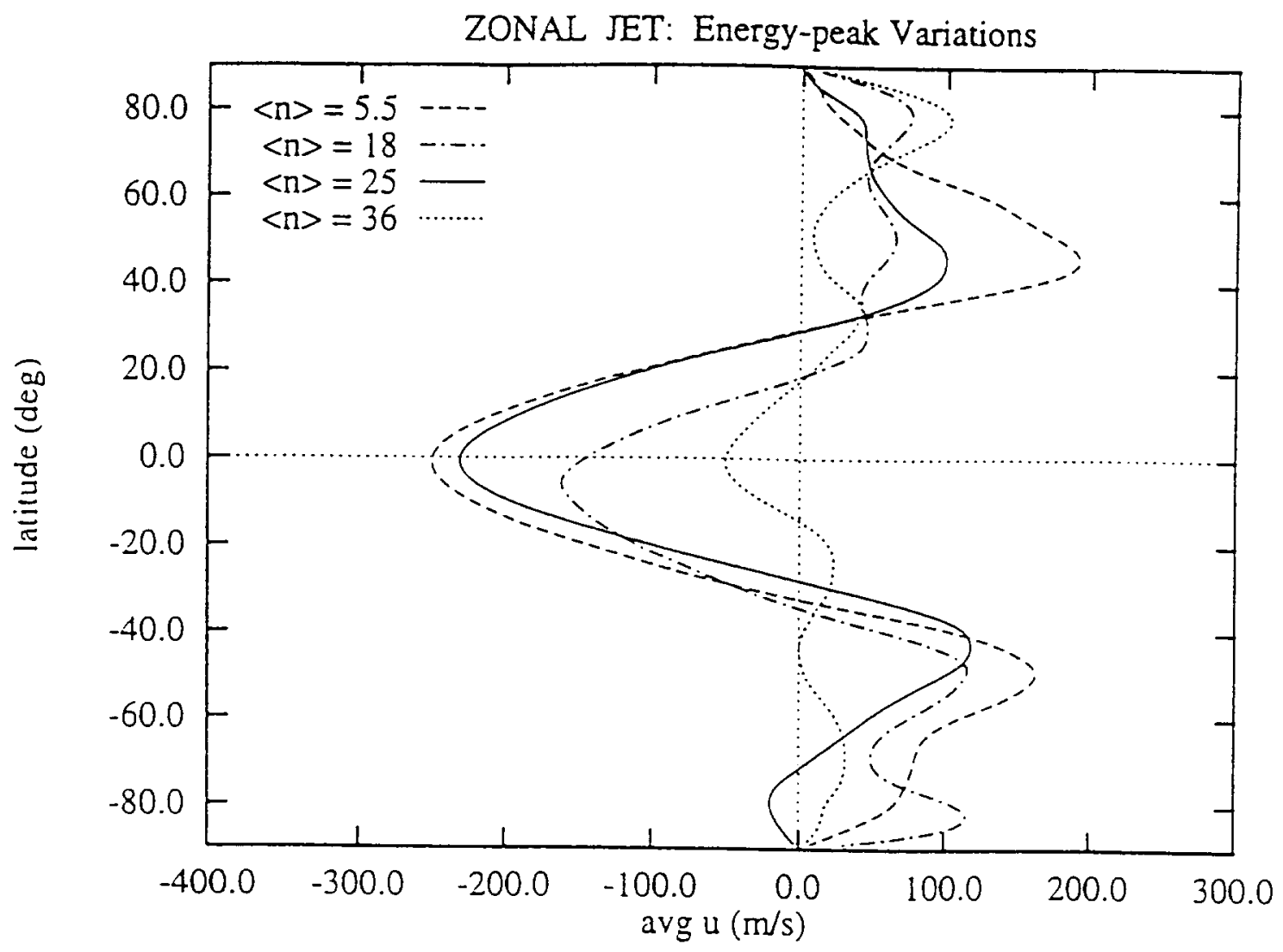

Figure 3: The zonal jet profile resulting from variations in the location of the energy peak, which is quantified by the energy centroid (see Runs N1 to N4). 


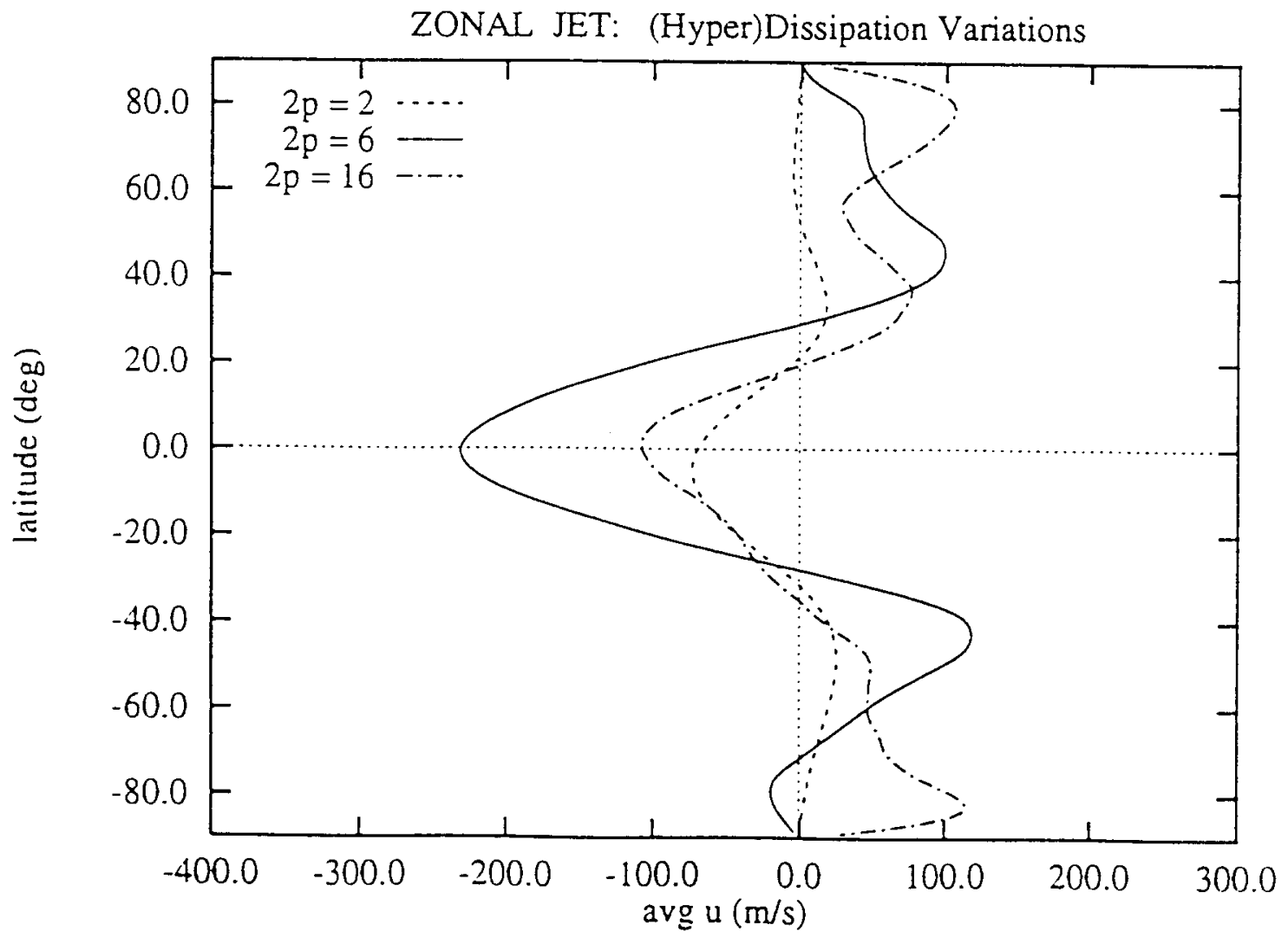

Figure 4: The zonal jet profile resulting from employing different (hyper)dissipation operators (Runs H1 to H3). $2 p$ is the power of the dissipation operator (i.e., 2 corresponds to normal molecular viscosity). 


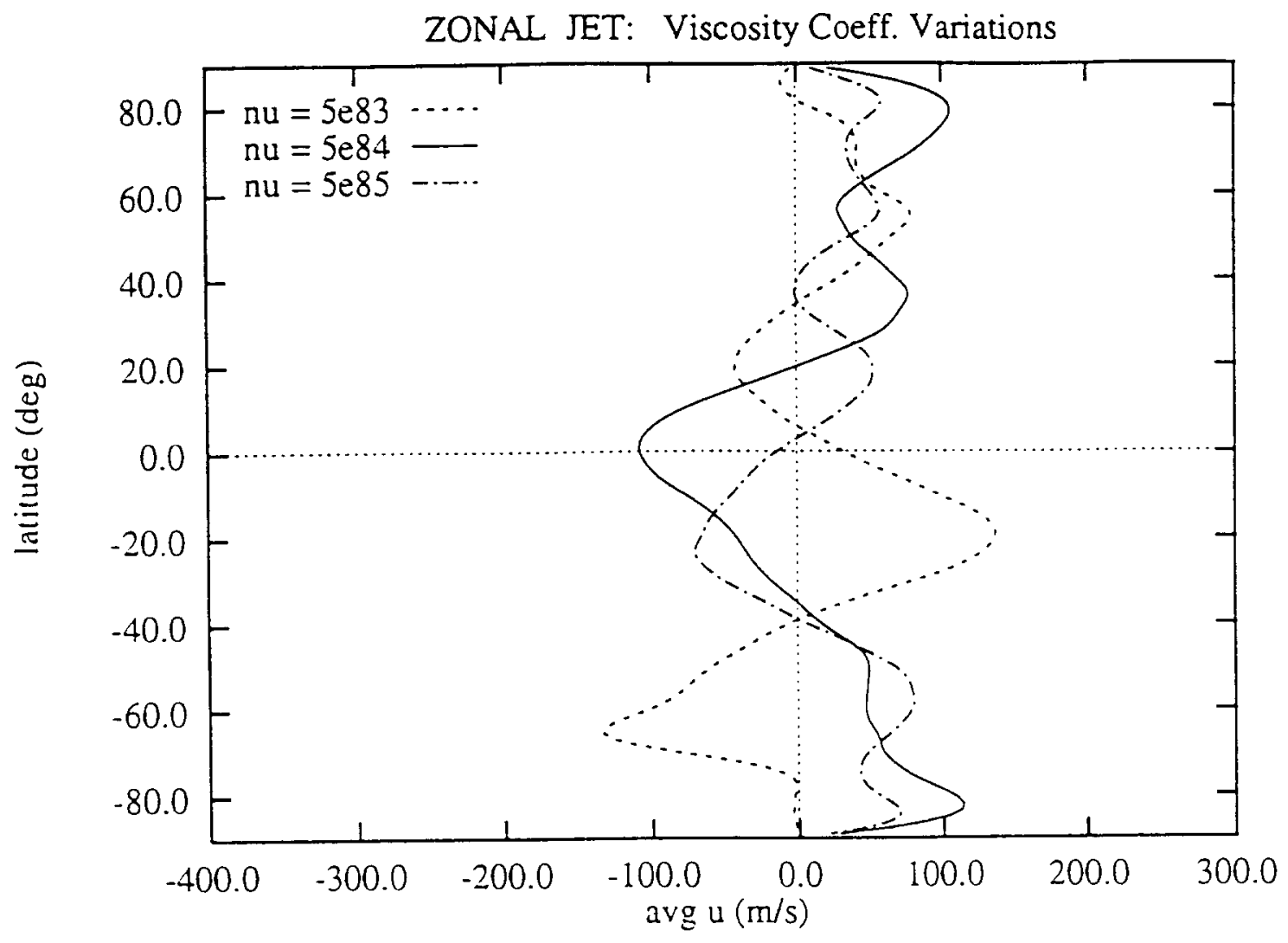

Figure 5: The zonal jet profile resulting from varying the viscosity coefficient with fixed, $2 p=16$ hyperdissipation operator (see Runs V1 to V3). Below the smallest value of the coefficient shown, the run is unstable. 


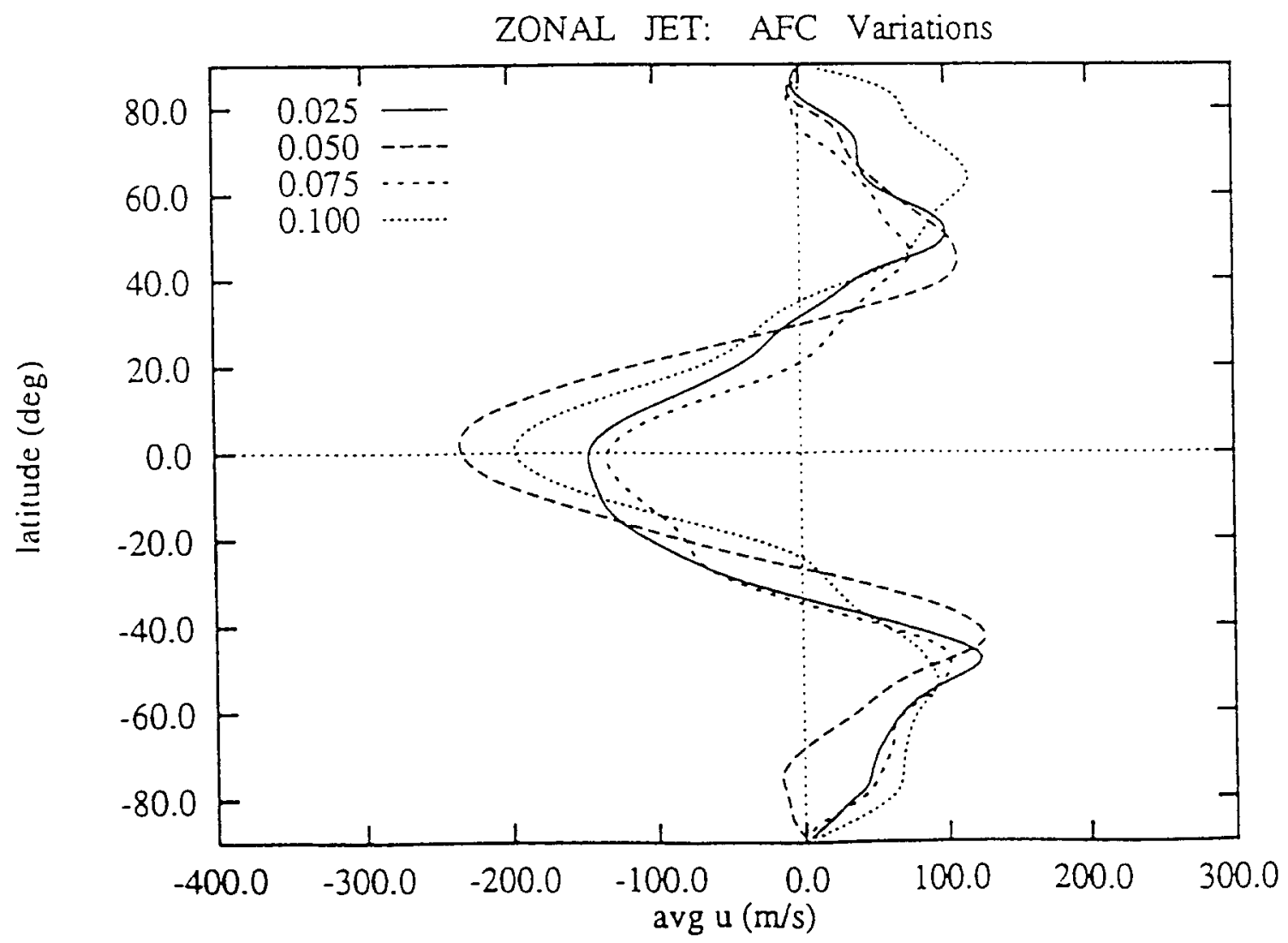

Figure 6: The zonal jet profile resulting from varying the Robert-Asselin filter coefficient with fixed, $2 p=6$ hyperdissipation operator (Runs A1 to A4 in Table 1). Below the smallest value of the coefficient shown, the run is unstable. 


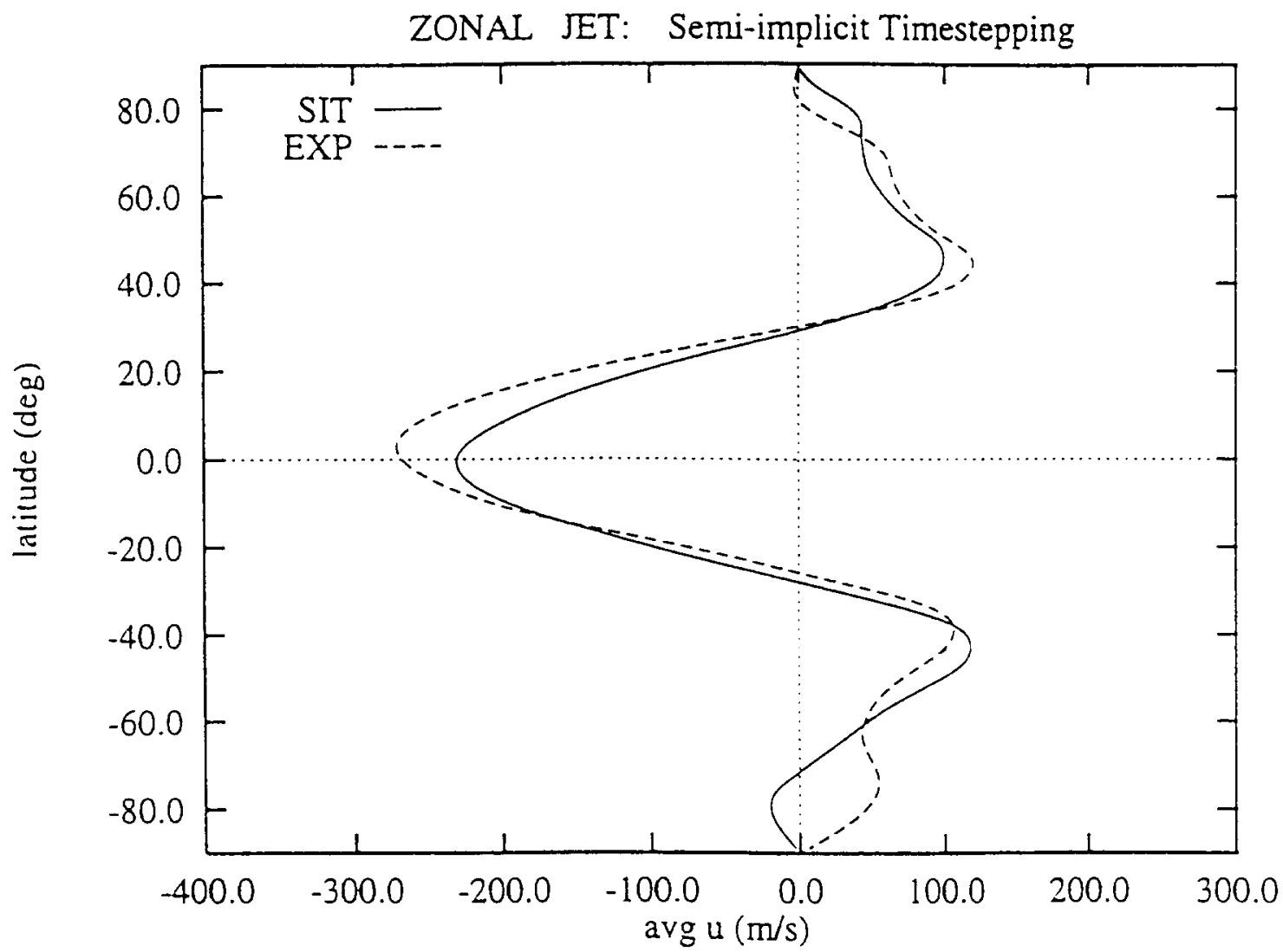

Figure 7: The zonal jet profile showing the effect of using a standard semi-implicit scheme (Runs $\mathrm{S} 1$ and S2). Due to the strong ageostrophic flow, the timestep sizes in both cases are equal. 


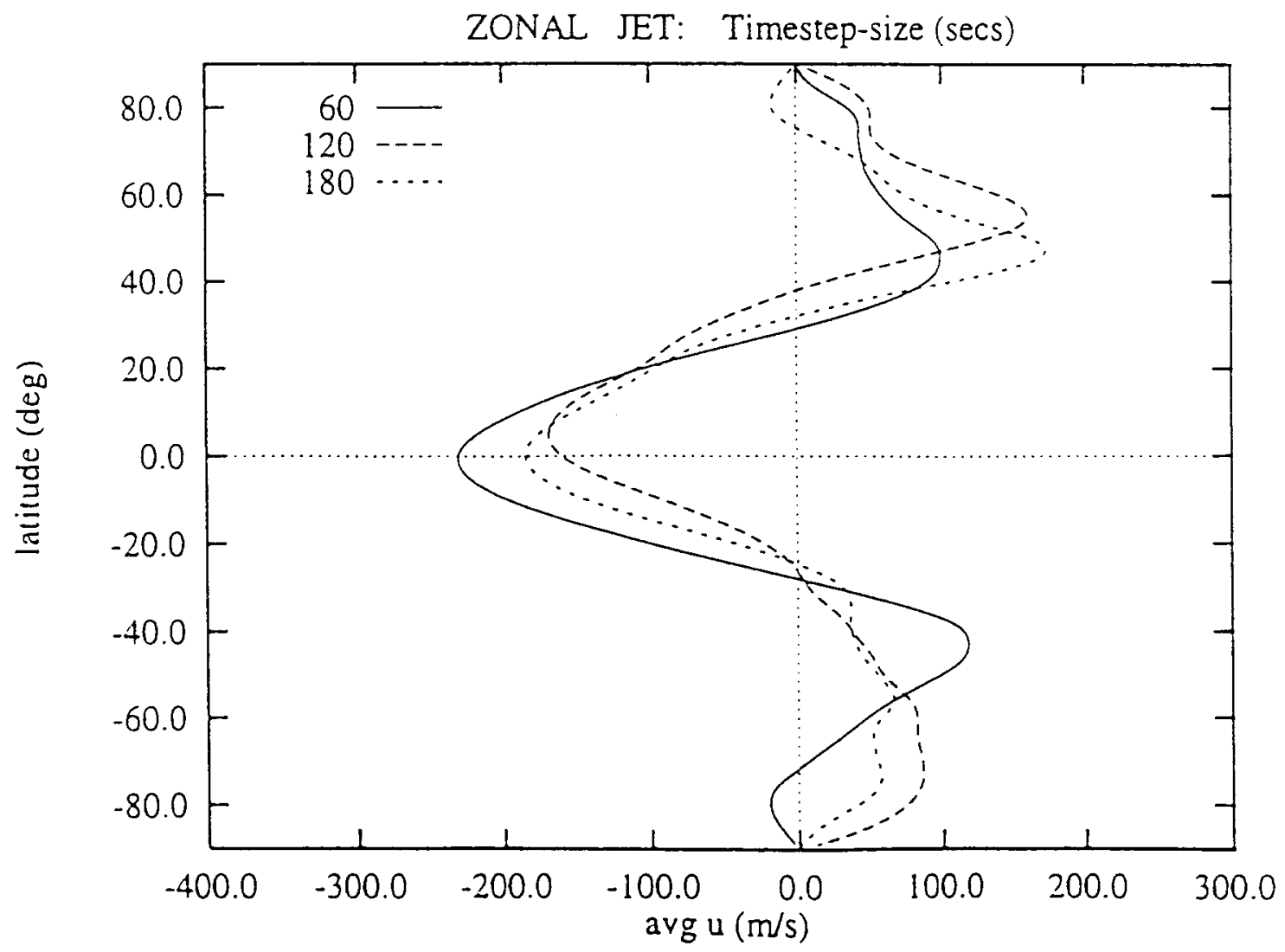

Figure 8: The zonal jet profile showing the effect of choosing three different timestep sizes (see Runs D1 to D3 in Table 1). Above the largest size shown, the run is unstable. 